\title{
Speaking up against inequity and racism
}

\author{
Inequalities are prevalent across the spectrum of cancer research and patient care, with destructive repercussions \\ for people and society. We cannot ignore them and must act against the social injustices that perpetuate them.
}

"In the end, we will remember not the words of our enemies, but the silence of our friends." Martin Luther King Jr.

That science, research and medicine strive for the benefit of humankind is a truism. That they are the products of the societies in which they operate, and thus reflect the privileges and shortcomings of those societies, is also a fact - one that often remains in the background of scientific discourse. The unvarnished reality is that inequalities cut deeply through biomedical science and patient care, with a devastating impact on individuals and communities.

The cancer field is not immune to such disparities. Racial, ethnic and socioeconomic factors weigh heavily on cancer prevention, diagnosis, access to and quality of care and, ultimately, outcomes. In the USA, members of minority racial and ethnic groups suffer disproportionately from cancer. This is well documented for many affected communities, including Black Americans, who experience higher cancer mortality rates ${ }^{1}$ than those of the white population. For colorectal, prostate and female breast cancer in particular, both incidence and mortality are higher for Black people ${ }^{1}$. Black patients also have lower participation in clinical trials, even when these are testing treatments for cancer types that are highly prevalent in their population. As a result, they are denied access to potentially life-extending therapies, and clinical findings become skewed toward a non-representative, white majority. Race is a social construct that does not bear a clear relationship to genetics and biology. However, the lack of a sampling diversity that corresponds to the real-world population remains a pervasive concern in research, as it can obscure links with disease traits and therapy response and thereby reinforce health disparities ${ }^{2}$, as highlighted in the field of human genomics ${ }^{3}$.

The lack of appropriate representation is also reflected in the composition of the medical workforce, with only $5 \%$ of physicians and $2.3 \%$ of oncologists self-identifying as Black or African American, despite the fact that Black Americans make up $13.4 \%$ of the US population. Similarly, minority ethnic or racial groups reportedly comprise only $3-7 \%$ of biomedical research faculty in the USA ${ }^{4}$, even though they are better represented at the doctoral and postdoctoral levels.

The causes of these disparities are complex and include historical and structural racism, implicit racial and social biases, entrenched economic, educational and healthcare inequities, and the cultural and behavioral trends of communities and individuals. When considering these latter behavioral factors, it is important to acknowledge the mistrust of many African Americans toward the medical system in light of the exploitation and discrimination to which they have been subjected historically. The notorious Tuskegee syphilis study is one such example, as is the case of Henrietta Lacks in the cancer field - a story that is as much about the general lack of bioethical standards at the time as it is about suffering and dying of cancer in the racially segregated US society of the 1950s.

The scientific community has been working toward understanding and addressing these issues. In the USA, the National Institutes of Health (NIH) have a long-held policy on the inclusion of minorities in clinical research. The more recent launch of the NIH-driven All of Us research program also aims to engage participants from traditionally underrepresented groups so that contributed health data will be representative of the diversity in the US population ${ }^{5}$. Among other efforts by the American Association for Cancer Research, the 2020 by 2020 initiative aims to address cancer disparities in the African-American population by collecting genomic and clinical data from 2020 African-American patients with cancer. The US National Cancer Institute's Center to Reduce Cancer Health Disparities is dedicated to decreasing the disproportionate cancer burden in society through research, training, education and mentoring efforts. The American Society of Clinical Oncology has announced a strategic plan to increase racial and ethnic diversity in the oncology workforce. On a political level, policies such as the Affordable Care Act have enhanced healthcare equity by expanding coverage and access to medical care to disadvantaged groups, including Black people ${ }^{6}$. Affirmative changes in policy could in fact drive rapid and meaningful change. It has been estimated that $22 \%$ of the cancer deaths in 2018 in the USA could have been avoided had these patients had access to and quality of health care and treatment similar to that of college-educated people ${ }^{7}$.

Such efforts are important, but they are not yet enough. From access to diagnosis, treatment and care, to population representation in the patient cohorts that inform research findings and drive clinical discovery, racial and ethnic minorities remain disadvantaged around the world. The COVID-19 pandemic has shone new light on these health disparities. Although race and ethnographic data continue to be limited, emerging analyses show that in the USA, minority communities, including African Americans and Latino Americans, bear an unequal burden when it comes to infection and mortality rates $^{8}$. The underlying reasons await detailed study, but the fact that historical and systemic inequality and discrimination have been affecting these communities in terms of financial means, access to healthcare insurance and diagnostic and treatment centers, quality and security of housing, and their ability to avoid virus exposure through work, cannot be denied.

Against this backdrop of health inequity, rooted in large part in racial and social exclusion and discrimination, came the recent killing of George Floyd, an African American man, at the hands of police. This was not an isolated or unprecedented incident but rather one event in a long string of injustice and brutality due to racial discrimination in the USA. At the time of writing, on the day of George Floyd's Minneapolis memorial service, the words of the poet Paul Laurence Dunbar come to mind: "a pain still throbs in the old, old scars / and they pulse again with a keener sting." Systematic and institutionalized racism continues to ripple through society, and its corrosive effects cannot be ignored. Inequality and discrimination have many incarnations, but one of their common drivers is the neutrality of a majority, the indifference and passive acceptance that perpetuates injustice. The street protests that have swept through the USA during the past ten days have reignited a much-needed public dialog about racism. Biomedical science has an important part to play in this discussion. As nations reopen their economies and the research enterprise gears up again, we should not seek to 
restart, picking up from where we left off and returning to our own definition of normality. Rather, we should redouble efforts to alter the conditions that permit inequity to persist in academia and the industry, in healthcare systems and clinical practice, and in science communication and publishing.

How do we catalyze this change? How do we transform the heartfelt expressions of solidarity into something more tangible than words and more meaningful than symbolic gestures of inclusivity? A first, essential step is to give the voices that are being drowned out the space, attention and respect they are due. We need to listen, learn and reflect. Moreover, beyond denouncing the overt, poisonous hate that sustains racism, we need to talk about the latent intolerance, the implicit biases, the passive neutrality and the entrenched privilege that permit racism to persist. They are more difficult to discern and therefore are the hardest to uproot. Recognizing and addressing their presence in our daily lives and the social and professional structures in which we operate is essential. It may also be uncomfortable, but that is a good thing. Change does not come from a place of comfort. We must remember and act on this long after the street protests end and the news cycle moves on.

Nature Cancer stands with the Black community and minority and underrepresented groups against discrimination and intolerance. We are committed to supporting work on health disparities and diversity and highlighting these issues through our pages. We pledge to increase diversity in our reviewer pool and to amplify the voices of underrepresented minority authors. Finally, we promise to continue educating ourselves, so that we may contribute to the efforts to level inequalities in a meaningful manner. To that end, we welcome the comments and ideas of our readers at cancer@nature.com.

Published online: 16 June 2020

https://doi.org/10.1038/s43018-020-0091-x

References

1. Howlader, N. et al. (eds). SEER Cancer Statistics Review, 19752017 (National Cancer Institute, 2020); https://seer.cancer.gov/ csr/1975 2017/

2. Popejoy, A. B. \& Fullerton, S. M. Nature 538, 161-164 (2016).

3. Hindorff, L. et al. Nat. Rev. Genet. 19, 175-185 (2018).

4. Meyers, L. C., Brown, A. M., Moneta-Koehler, L. \& Chalkley, R. PLoS One https://doi.org/10.1371/journal.pone.0190606 (2018).

5. The All of Us Research Program Investigators. N. Engl. J. Med. 381, 668-676 (2019).

6. Gaffney, A. \& McCormick, D. Lancet 389, 1442-1452 (2017).

7. Polite, B. N., Gluck, Abbe, R. \& Brawley, OtisW. J. Am. Med. Assoc. 321, 1663-1664 (2019).

8. Hooper, M. W., Nápoles, A. M. \& Pérez-Stable, E. J. J. Am. Med. Assoc. https://doi.org/10.1001/jama.2020.8598 (2020). 